\title{
EMI-free Bidirectional Real-time Indoor Environment Monitoring System
}

\author{
Ngoc Quan Pham ${ }^{1}$, Vega Pradana Rachim ${ }^{1}$, Jinyoung An ${ }^{1}$, Wan-Young Chung ${ }^{1}$ \\ ${ }^{1}$ Pukyong National University, Busan 608-737, South Korea \\ wychung@pknu.ac.kr
}

\begin{abstract}
Indoor air quality, temperature, and relative humidity are broadly referred to the environmental characteristics inside buildings that may affect a thermal comfort and healthy life of the personnel. Thus, monitoring continuously these parameters is essential in order to understand and prevent air pollution, preserve health and improve labor productivity. Therefore, we propose a unique indoor environmental monitoring system which implemented EMI-free visible light communication (VLC) technology. Our system included a base station integrated into the LED lamp, sensor modules placed in regular breathing zone at the $1.5 \mathrm{~m}$ height of workplace. Five vital parameters of indoor environment in multi-location are being remotely monitoring in real-time. After collecting the sensing data from electrochemical sensors, we modulated these data into the light beam by LED and photodiode are employed as the VLC receiver with high data rate. Our average-voltage tracking algorithm is also adapted to allow the LED illumination to utilize feasibly for dual purposes as a lighting system and a bidirectional communication in long range. Finally, the measured data are displayed by using a user interface in real-time.
\end{abstract}

Key words: indoor air quality, environmental monitoring, electrochemical sensor, VLC.

\section{Introduction}

While most individuals perceive that outdoor air quality plays an important role in their health quality, many of people are unaware a variety of health risks which is potentially affected by indoor air pollution. According to the Environmental Protection Agency (EPA) studies, indoor air may be referred two to five times and sometimes up to 100 times hazard than outdoor air in term of pollutant level. These levels of indoor air pollutants are of particular concern because people spend approximately 90 percent of their time indoors [1]. In this study, we developed a unique indoor environmental monitoring to guarantee the comfortable and healthy environment. The concentration of a broad gas range is measured by oxidizing or reducing the target gas at a sensor electrode of and measuring the resulting current, including $\mathrm{O}_{2}$ concentration, $\mathrm{CO}_{2}$ concentration and volatile organic compounds (VOCs) which are from paints, cleaning supplies, furnishings, office equipment, etc.

Besides, most of the indoor environmental monitoring research utilized the radio frequency (RF) as a wireless communication medium to carry information. However, long-term exposure to RFs could lead to degrade human health by the detrimental biological effects on the human body. Consequently, these negative side effects attribute to the opposite of the original purpose behind the indoor environmental monitoring system which produces a healthy environment. As a potential alternative for the existing RF, visible light communication (VLC) technology acts as a solution to overcome these advantages. VLC is one of the free-space optics technology that uses visible light beams as the variant in data communication. The harmless and EMI-free characteristics of VLC not only provide a better long-term technique for indoor environmental monitoring but also support the possibility of integrated wireless communication in RF-limited areas. This paper introduces a real-time environmental monitoring system which adapts an average-voltage tracking algorithm-based VLC technology in a practical indoor environment.

\section{System Design}

Fig.1 shows the block diagram of the sensor module, including the sensor and processing unit. In our proposed system, the LEDs and photodiode are employed as the transmitting and receiving elements, respectively. Three 


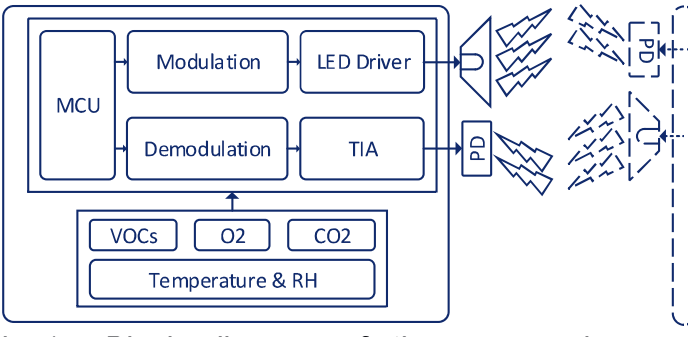

Fig. 1. Block diagram of the proposed sensor module.

sensors (HDC1080, CCS811 and Grove - Gas Sensor) are programmed in each node. Whenever the monitoring server needs the environmental information, a relevant command is sent from the base station to each sensor module through VLC. After collecting and processing data, a microcontroller integrated in each node will modulate environmental information into the light beam for transmitting back to the base station.

Our scenario is depicted in Fig.2, illustrating simply our proposed ideal; the base station attached in the ceiling is connected to database server, two sensor modules collect a variety of environmental data. Moreover, our averagevoltage tracking algorithm [2] is implemented to eliminate the ambient noise dominated by lighting system.

\section{Experimental Results and Conclusion}

An experiment is carried out from Friday to Saturday with totally the period of 48 hours in laboratory environment. With a long distance of $5 \mathrm{~m}$, error-free is achieved without lens and the data-rate is up to $1 \mathrm{Mbps}$, resulting in the possible of real-time indoor monitoring system.

Fig. 3 shows data collected from sensor module. On the first day, 12 persons worked during office hours, there were 4 persons on the second day. While the number of people was
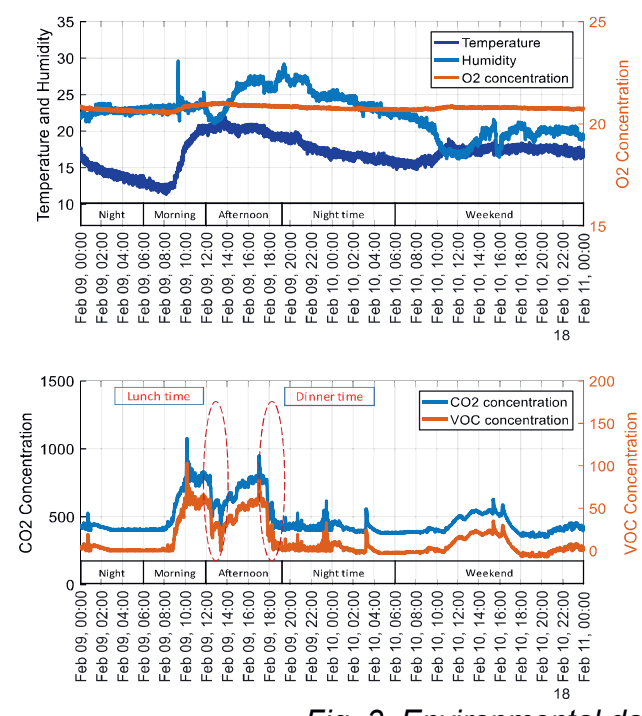

Fig. 3. Environmental data of laboratory within 2 days.

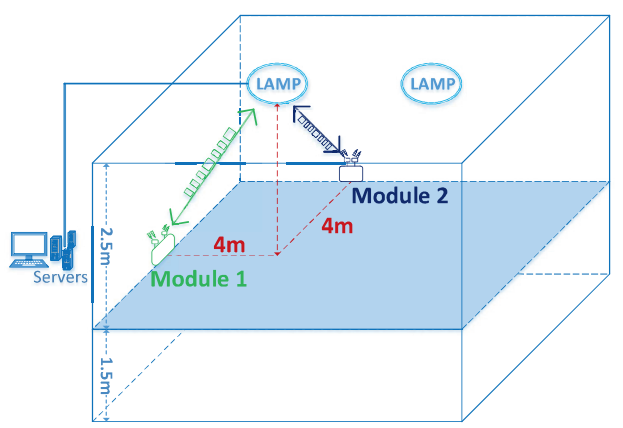

Fig. 2. Testbed architecture of the bidirectional environmental monitoring system.

increasing in workday, $\mathrm{CO}_{2}$ and VOCs levels were also witnessed a significant raise, leading to bad quality range, but still under acceptable levels. In contrast, these figures decreased before maintaining stably at the healthy range in weekend or night time. Furthermore, the lunch and dinner time were experienced the sudden drop. Meanwhile there was no remarkable change in $\mathrm{O}_{2}$ concentration, the temperature was low at midnight, decreasing in the early hours of the morning, then increasing rapidly until just after midday.

The experiments also figure out that indoor air quality could drop unconsciously into adverse range, this tends to cause a long-term consequence for human being. Therefore, it is necessary to monitor indoor environment. The harmless proposed system is useful to contribute a healthy environment.

\section{References}

[1] World Health Organization (WHO. "WHO guidelines for indoor air quality: selected pollutants." (2010).

[2] Pham, Q. N., Rachim, V. P., An, J., \& Chung, W. Y. Ambient Light Rejection Using a Novel Average Voltage Tracking in VLC System. Applied Sciences, 7(7), 670. (2017).
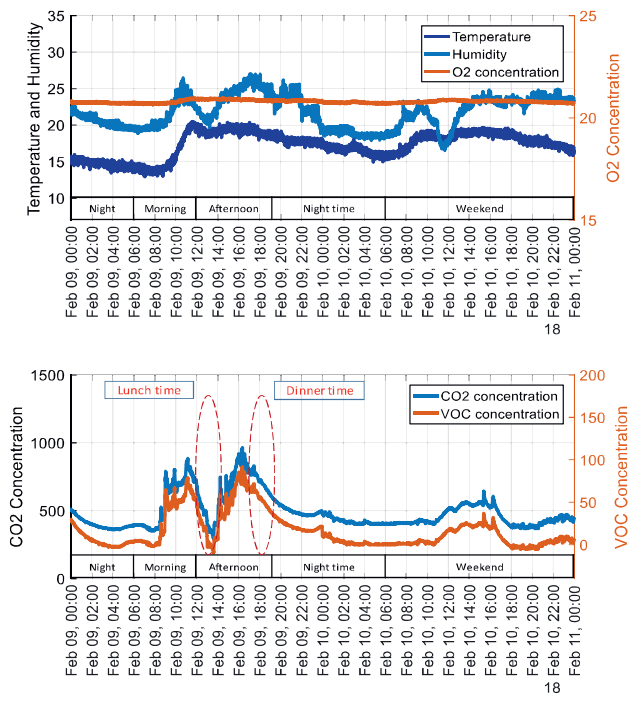